\title{
1 The evolution of siderophore production as a competitive
}

2 trait

3

4 Abstract

5 Microbes have the potential to be highly cooperative organisms. The archetype of microbial

6 cooperation is often considered to be the secretion of siderophores, molecules scavenging

7 iron, where cooperation is threatened by "cheater" genotypes that use siderophores without

8 making them. Here we show that this view neglects a key piece of biology: siderophores are

9 imported by specific receptors that constrain their use by competing strains. We study the

10 effect of this specificity in an eco-evolutionary model, in which we vary siderophore sharing

11 between strains, and compare fully shared siderophores with private siderophores. Privatising

12 siderophores fundamentally alters their evolution. Rather than a canonical cooperative good,

13 siderophores become a competitive trait used to pillage iron from other strains. We also study

14 the physiological regulation of siderophores using in-silico long-term evolution. While shared siderophores evolve to be downregulated in the presence of a competitor, as expected for a cooperative trait, privatised siderophores evolve to be upregulated. We evaluate these

17 predictions using published data suggesting that some siderophores are upregulated in response to competition akin to competitive traits like antibiotics. While siderophores can act as a cooperative good for single genotypes, therefore, we argue that their role in competition is fundamental to understanding their biology. 
Main text

27 Iron limits the growth of many microorganisms making it a key determinant of evolutionary fitness and ecological competition. To cope with iron limitation, microbes secrete siderophores into the environment (Ratledge and Dover 2000; Chakraborty et al. 2013). These molecules chelate insoluble iron and allow it to be taken up via siderophore receptors (Wandersman and Delepelaire 2004). Cells of one genotype (strain) have matching siderophores and siderophore receptors such that the siderophores produced by a cell are shared with other cells of the same strain (Griffin et al. 2004). Because siderophores can also carry metabolic costs (Griffin et al. 2004), siderophores have been identified as a microbial public good (West et al. 2006, 2007; Nadell et al. 2009), with the key corollary that a nonproducer (“cheater") mutant may outcompete a producer by using its siderophores without paying the production cost (Griffin et al. 2004; Ross-Gillespie et al. 2007; Brown et al. 2009). While it is clear that siderophores have the potential to act as public goods between cells of a single genotype, this perspective lacks a key piece of siderophore biology. The different strains and species that commonly meet in natural communities possess a large diversity of both siderophores and siderophore receptors (Miethke and Marahiel 2007; Hider and Kong 2010) and many receptors bind siderophores in a highly specific manner (Braun 2001; Hantke 2001). Experiments have shown that this specificity can greatly limit siderophore cross-feeding between competing strains (Joshi et al. 2006; Khan et al. 2006). Siderophores may then act as public goods within a strain but they can be private goods between different strains (Joshi et al. 2006). This privatisation is further amplified when bacteria grow in clonal patches, which is common in many environments (Mitri et al. 2015; 
close to the strain that released them (Nadell et al. 2010; Julou et al. 2013; Kümmerli et al. 2014; Oliveira et al. 2014).

How does siderophore privatisation affect its social role and the evolution of siderophore production? To answer these questions, we developed a new eco-evolutionary model of siderophore production. Our theory is centred upon an explicit mechanistic model of siderophore-scavenging (Fgaier and Eberl 2010; Lee et al. 2016), which we extend to allow different levels of inter-strain sharing of siderophores. When siderophores are private, or partially private, we find they evolve as an exploitative strategy that functions to steal iron from competitors. Moreover, our model predicts that privatisation leads to a major shift in the regulation of siderophore production. While public siderophores are downregulated in the presence of competitors, partly privatised siderophores evolve to be upregulated. We use published data to test between these two regulatory responses and argue that the role of siderophores in ecological competition is fundamental to both their evolution and regulation. Materials and Methods Model Overview

Our goal is to understand how siderophore privatisation affects the evolution of siderophore

66 production and regulation. The core of our approach is based upon the biochemical mechanisms of iron scavenging via siderophores, a well-studied process that includes secretion of siderophore molecules, their binding to iron and subsequent formation of siderophore-iron complexes, the uptake of these complexes via siderophore receptors, and the loss of siderophores through diffusion (Winkelmann et al. 1987; Winkelmann 1991; Andrews et al. 2003). All of these processes affect the evolutionary costs and benefits of siderophore production (Kümmerli et al. 2009a, 2014; Lee et al. 2016) and we model the processes explicitly using ordinary differential equations (ODEs). While this leads to relatively large 
systems of equations, this allows us to make full use of the detailed experimental work on siderophore production (Boukhalfa and Crumbliss 2002; Mey et al. 2004; Hider and Kong 2010). In addition, as we will show, the relative complexity that comes with this realism does not prevent us from extracting clear and testable predictions from our model. We embed the model within an implicit meta-population framework (Cremer et al. 2012; Oliveira et al. 2014), where we study sets of strains that grow, interact and compete over iron in local patches before dispersing and seeding new patches. With this, we can study the evolutionary fate of strains that differ in their siderophore production as a function of the ecology and, importantly, the level of privatisation of siderophores that limits their use to a single strain.

\section{Local dynamics}

We study strains that migrate to and interact in a focal patch, which could represent for example a small neighbourhood within a structured community, or a host organism. Most theory to date has focussed on the interaction between producers and non-producers (West and Buckling 2003; Eberl and Collinson 2009; Inglis et al. 2011). However, in our model all strains have the potential to produce siderophores, although they may evolve not to produce any. We study selection on the investment into siderophore production $(f)$, which can take any value in the range $[0,1]$, where 0 corresponds to non-production of siderophores. The number of different strains that interact in a single patch is given by $n(n=1,2,3, \ldots)$. This number determines the strength of ecological competition in the patch: when a single strain seeds a patch $(n=1)$ there is no competition between genotypes; when two or more strains seed a patch, there is inter-strain competition for iron; and this competition increases with the number of competing strains.

Each strain in the focal patch is a distinct genotype that originates from the external ecological landscape. While bacterial genotypes have the potential to produce multiple 
100 Dumas et al. 2013), we follow previous models (West and Buckling 2003; Lee et al. 2012)

101 and assume that each strain can produce at most one siderophore. Each strain expresses also

102 the cognate receptor for its siderophore but may also take up siderophores produced by other

103 strains, either because its receptor has affinity for other siderophores (Crowley et al. 1991) or

104 because it co-expresses multiple siderophore receptors (Cornelis and Matthijs 2002; Cornelis and Bodilis 2009). We make this between-strain sharing a tuneable parameter $(s \in[0,1])$ of our model, where $s=0$ means that only the producer strain benefits and $s=1$ means that all

107 strains benefit equally.

108

109

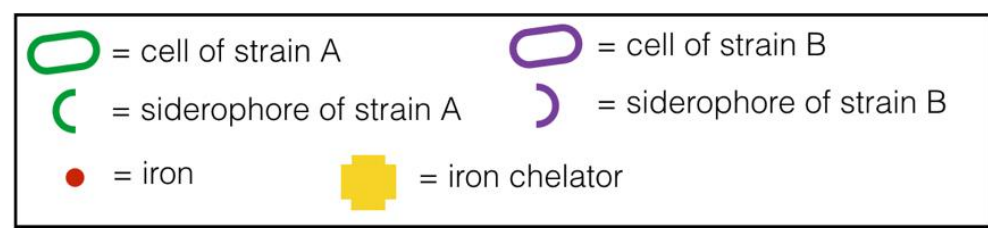

Scavenging process (model parameters)
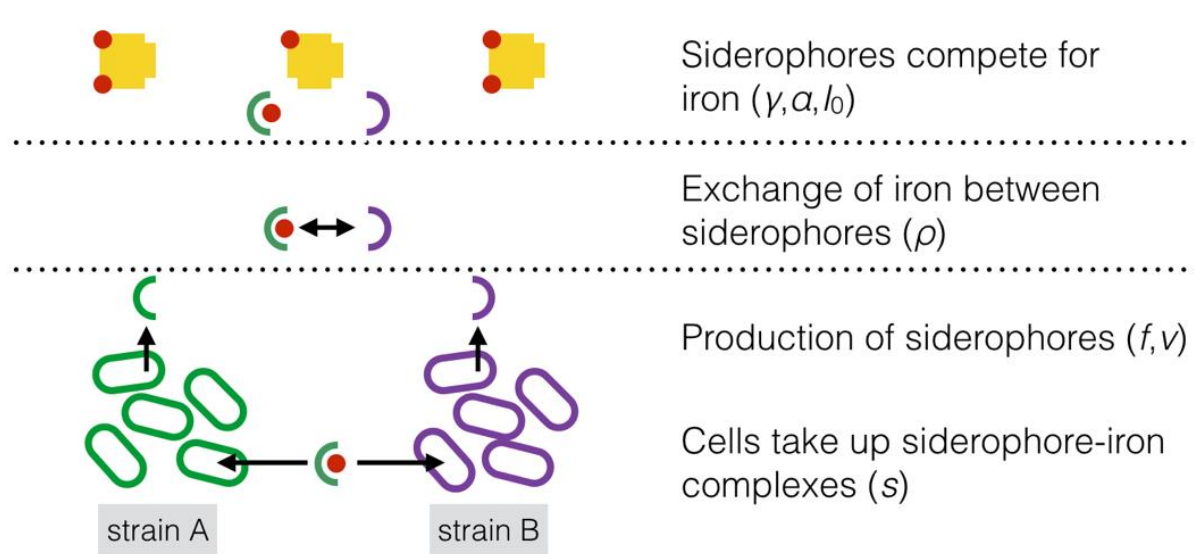

111 Figure 1 | A schematic view of siderophore scavenging divided into its key component processes.

112 Iron scavenging begins with the secretion of siderophores by cells with a specified level of investment 113 and yield. Siderophores (half-circles) then bind the environmental iron (red dots on yellow shapes, 114 representing for example other iron chelators), that is present in the patch according to its reflux rate

$115(\alpha)$ and its concentration in the external environment $\left(I_{0}\right)$. Siderophores and iron form complexes

116 according to the siderophore affinity $\gamma$. Siderophores can also 'steal' or strip away iron from existing 117 complexes at a rate that depends on the iron exchange parameter $\rho$. Finally, different strains compete 118 for the uptake of siderophore-iron complexes according to the extent to which siderophores are shared 119 between strains $(s)$. For clarity, each process is shown separately in this cartoon, but in our model, all 120 processes occur continuously in one well-mixed patch. 
121 Siderophores are secreted molecules and iron scavenging occurs outside the cells through several processes. For clarity, we capture scavenging as four key processes, which we incorporate into our model to occur simultaneously (Figure 1):

1) Siderophore secretion: we assume the production of siderophores to be metabolically costly (Griffin et al. 2004) and to depend on the level of energy invested (f), which is traded-off against the energy allocated to growth (Lee et al. 2016). The total amount of energy available to the cells depends on the uptake of a non-limiting nutrient $(N)$ which is

2) Binding iron: Excreted siderophores bind the environmental iron with a certain affinity $(\gamma)$, thereby making this iron unavailable for other siderophores (Boukhalfa and Crumbliss 2002; Hider and Kong 2010).

3) Stealing iron: Siderophores can strip away iron from other siderophore-iron complexes with rate $\rho$. The extent of this exchange will depend on the quantity and the affinity of the siderophore that is stealing the iron and the quantity of iron-complexes by other

4) Cellular uptake: Cells take up their own type of siderophore-iron complexes via siderophore receptors. And strains may, as discussed above, also be able to uptake siderophores from other strains (Joshi et al. 2006; Khan et al. 2006). For simplicity, we do not explicitly model siderophore diversity and receptor affinities, but we capture the between-strain sharing through a single parameter $s(s \in[0,1])$, which gives the fraction of a siderophore concentration that can be used by strains other than the producer strain. We also assume that cells carry a limited amount of siderophore receptors, giving a saturating siderophore uptake response. Siderophore sharing may also be affected by spatial arrangement of the different bacterial strains (Nadell et al. 2010; Julou et al. 2013; 
Kümmerli et al. 2014). While there is the potential to extend our model to capture such effects explicitly, we decide to focus here on a well-mixed case.

148 We can then capture the dynamics of siderophore scavenging within a single patch for a

149 single-strain $(n=1)$ by the ODE system

$$
\left\{\begin{array}{c}
\frac{d C(t)}{d t}=\mu \frac{(1-f)}{(1-f)+\beta}\left(\frac{P(t)}{P(t)+K_{P}}+\epsilon I(t)\right) C(t)-d_{C} C(t) \\
\frac{d S(t)}{d t}=v f C(t)+\frac{(1-f)}{(1-f)+\beta} \frac{P(t)}{P(t)+K_{P}} C(t)-\gamma S(t) I(t)-d_{S} S(t) \\
\frac{d P(t)}{d t}=\gamma S(t) I(t)-\frac{(1-f)}{(1-f)+\beta} \frac{P(t)}{P(t)+K_{P}} C(t)-d_{P} P(t) \\
\frac{d I(t)}{d t}=a\left(I_{0}-I(t)\right)-\gamma S(t) I(t)-\epsilon C(t) I(t)
\end{array}\right.
$$

152

153

154

where $C(t)$ is the cell density of the focal strain, $S(t)$ is the concentration of its siderophores, $P(t)$ is the concentration of iron-siderophore complex, and $I(t)$ is the concentration of available iron at time $t$. We model cellular metabolism such that cellular growth relies both on iron and on other sources of energy, such as carbohydrates, lipids or protein. Iron is often limiting due to low concentrations of its soluble form (Wandersman and Delepelaire 2004), and we assume that other energy sources are abundant. From these assumptions, we show in the Supplementary Materials and Methods how we can go from a form where non-iron nutrients are explicitly captured to Equation (1) where these nutrients are captured implicitly. We also assume that the production of siderophores does not require significant iron, which is consistent with their chemistry (Hider and Kong 2010). 
166 Cells proliferate with a rate that depends on a maximum growth rate $\mu$, the investment of

167 energy into cell growth, and the iron available per cell. The available iron depends on the 168 uptake of iron-siderophore complex $(P)$ and iron-uptake through siderophore-independent 169 mechanisms $(\varepsilon)$ (Wandersman and Delepelaire 2004). Cells invest a fraction $1-f$ of their 170 energy into building up cell biomass, and we assume here the presence of an abundant energy source that is captured in $\beta$ of the Monod function with saturation constant $K$ (see Supplementary Materials and Methods). The acquisition of iron through uptake of siderophore-iron complexes also follows a Monod function with constant $K_{\mathrm{P}}$. Cells die at a constant per capita rate $d_{\mathrm{C}}$. The free siderophore concentration $S(t)$ changes over time due to

175 cellular siderophore production, which is proportional to the amount of energy that the cells invest into siderophore production $(f)$ and to the siderophore production yield $(v)$. We assume that the siderophores are instantaneously recycled from iron-siderophore complexes: the rate of siderophore recycling is equal to the rate of iron-siderophore complex uptake. And

179 siderophores are lost from the system through diffusion $\left(d_{\mathrm{S}}\right)$.

180 Our model follows chemostat dynamics with a constant input of iron as well as loss of siderophores and siderophore-iron complexes. The external concentration of iron is $I_{0}$, and there is a reflux of iron into the patch at reflux rate $a$. The concentration of iron-bound siderophores changes over time due to the formation of such complexes (at a rate $\gamma$, that

184 represents the affinity of siderophore for iron), due to the uptake by cells, and due to loss

$185\left(d_{P}\right)$. Finally, the concentration of available iron follows chemostat dynamics with a reflux of external iron and depletion through the formation of iron-siderophore complexes.

187 When multiple strains interact in a patch $(n>1)$ we extend the number of equations accordingly. We illustrate this here with the extended system for two strains, to demonstrate the implementation of between-strain sharing of siderophores and well as of ligand exchange: 


$$
\left\{\begin{array}{c}
\frac{d C_{1}}{d t}=\mu \frac{\left(1-f_{1}\right)}{\left(1-f_{1}\right)+\beta}\left(\frac{P_{1}+s P_{2}}{P_{1}+s P_{2}+K_{P}}+\epsilon I\right) C_{1}-d_{C} C_{1} \\
\frac{d S_{1}}{d t}=v f_{1} C_{1}+\frac{\left(1-f_{1}\right)}{\left(1-f_{1}\right)+\beta} \frac{P_{1}+s P_{2}}{P_{1}+s P_{2}+K_{P}} C_{1}-\gamma S_{1} I-d_{S} S_{1}+\rho\left(S_{2} P_{1}-S_{1} P_{2}\right) \\
\frac{d P_{1}}{d t}=\gamma S_{1} I-\frac{\left(1-f_{1}\right)}{\left(1-f_{1}\right)+\beta} \frac{P_{1}}{P_{1}+s P_{2}+K_{P}} C_{1}-\frac{\left(1-f_{2}\right)}{\left(1-f_{2}\right)+\beta} \frac{s P_{1}}{P_{2}+s P_{1}+K_{P}} C_{2}-d_{P} P_{1} \\
\frac{d C_{2}}{d t}=\mu \frac{\left(1-f_{2}\right)}{\left(1-f_{2}\right)+\beta}\left(\frac{P_{2}+s P_{1}}{P_{2}+s P_{1}+K_{P}}+\epsilon I\right) C_{2}-d_{C} C_{2} \\
\frac{d S_{2}}{d t}=v f_{2} C_{2}+\frac{\left(1-f_{2}\right)}{\left(1-f_{2}\right)+\beta} \frac{P_{2}+s P_{1}}{P_{2}+s P_{1}+K_{P}} C_{2}-\gamma S_{2} I-d_{S} S_{2}+\rho\left(S_{1} P_{2}-S_{2} P_{1}\right) \\
\frac{\left(1-f_{2}\right)}{d t}=\gamma S_{2} I-\frac{P_{2}}{\left(1-f_{2}\right)+\beta} \frac{\left(1-f_{1}\right)}{P_{2}+s P_{1}+K_{P}} C_{2}-\frac{\left(1-f_{1}\right)+\beta}{P_{1}+s P_{2}+K_{P}} C_{1}-d_{P} P_{2} \\
\frac{d I}{d t}=a\left(S_{2} P_{1}-S_{1} P_{2}\right)
\end{array}\right.
$$

We assume that siderophores can strip away iron from other iron-siderophore complexes, according to the reactions $\mathrm{S}_{1}+\mathrm{P}_{2} \rightarrow \mathrm{S}_{2}+\mathrm{P}_{1}$ and $\mathrm{S}_{2}+\mathrm{P}_{1} \rightarrow \mathrm{S}_{1}+\mathrm{P}_{2}$, which proceed with mutual rate constant $\rho$. Importantly, the siderophore produced by a bacterial strain can to some extent also be used by other strains. The sharing parameter $s$ determines how much a siderophore can be used by the other strains that did not produce this siderophore. The number of strains $(n)$ gives the strength of ecological competition that is intensified with increasing number of strains.

We assume that the chemical properties of siderophores (amount of sharing, affinity, loss, yield) are identical for all interacting strains. In reality, siderophores can differ in their 
chemical properties (Cornelis and Matthijs 2002), but with this assumption we can study the isolated effect of siderophore privatisation on its evolution. Diversity in the other properties such as affinity and yield will have additional effects on siderophore evolution. To solve the system of ODEs we impose initial conditions $(C(t=0)=1, S(t=0)=0, P(t=0)=$ $\left.0, I(t=0)=I_{0}\right)$ and we use the finite difference method with constant grid spacing and implicit

210 Euler stepping with constant time steps to solve the equations numerically using the standard

211 ODE solver from GSL (Gnu Standard Library) in C++. We summarize the biological significance and default values of the parameters used in our model in the Supplementary Table 1 in Supplementary Materials and Methods.

\section{Physiological regulation of siderophores}

216 Our first models studied strains that invest a fixed proportion of their resources into

217 siderophores. We next study the evolution of regulated siderophore production. We replace

218 the fixed investment $f$ by a sigmoid, quasi-step function that represents a simple sensory

219 trigger function that responds to a signal $x$, give through

$$
f=f_{b a s}+\frac{\left(f_{a c t}-f_{b a s}\right)}{(1+\exp (100(x-T)))}
$$

Under this functional form, $f$ approximately takes the 'activated' value $f_{\text {act }}$ when the signal $x$ is above the threshold T, and the 'basal' value $f_{\text {bas }}$ otherwise. We consider three possible 
intracellular iron concentration, which is known to strongly regulate siderophore production in some species (Schmitt and Holmes 1991; Ratledge and Dover 2000; Rodriguez et al. 2002; Chakraborty et al. 2013), which will be proportional to the iron-siderophore complexes that a

231 focal strain $i$ can use, given as $\left[(1-s) P_{i}+s \sum_{j=1}^{n} P_{j}\right]$. The second source of information is clonemate density $\left(C_{\mathrm{i}}\right)$, which can be detected by quorum sensing or another product specific to the focal strain (Stintzi et al. 1998; Lewenza et al. 1999; Mok et al. 2003). Finally, we consider competitor cell density $\left(\left(\sum_{j=1}^{n} C_{j}-C_{i}\right)\right.$, which can be detected by any compound that is specific to the competitor. This detection might include non-self quorum sensing autoinducers but also sensing the damage from antibiotics or bacteriocins of the competing strain (competition sensing, Cornforth \& Foster 2013).

In our optimising algorithm, the three parameters that define the shape of the trigger function ( $f_{\text {act }}, f_{\text {bas }}$, and $T$ ) will initially be selected at random and be the same for all strains, and then we interactively test the invasion of a new strategy with either of the parameters changed. Note that while we use the term 'activated' for above threshold, the strains are free to evolve either an increase or decrease in the production of siderophores upon activation.

Meta-population dynamics

We embed our model of local competition between strains within a meta-population to study how different strategies evolve over time. Our meta-population model is based on previously published work and assumes an infinite number of local patches that are linked through the dynamics of a simple microbial life-cycle (Cremer et al. 2012; Oliveira et al. 2014):

1) Seeding: An empty patch is seeded with a certain number $n$ of different strains with initially small density $\left(C_{\mathrm{i}}(0)=1\right)$. The strategy for each strain is determined according to the frequency of the strategies in the entire meta-population. 
2) Competition: Strains grow and interact within each patch of the meta-population according to the local dynamics model given in equation (1) and (2). Interactions are simulated for a fixed amount of time (that can be then varied as a parameter).

3) Mixing: Cells from all patches disperse and mix, leading to a new seeding episode.

We then assess the evolutionary fate of new strategies that appear in the meta-population. To do this, we use invasion analysis, which is based upon the logic of evolutionary game theory (Maynard Smith 1982). When it can be used, invasion analysis is a powerful way to study coevolving strategies that allows one to avoid explicitly modelling each step in a life-cycle (Nowak and Sigmund 2004). Specifically, to follow the evolution of new siderophore production strategies, we study how a rare mutant or immigrant with the new strategy performs in a meta-population where all other strains perform another strategy. We can then ask whether this immigrant will successfully invade the resident strategy population, or instead go extinct.

We calculate invasion ability from the fitness of the new mutant strain ( $\left.w_{\text {inv }}\right)$ and the fitness of the resident strategy $\left(w_{\text {res }}\right)$. The fitness of the invader, since it is rare, is determined by its local competition with other strains that have the resident strategy, $w_{\text {inv }}=w\left(f_{\text {inv }} \mid f_{\text {res }}\right)$. The fitness of the resident strategy, which is very common and will therefore nearly always meet itself-is determined by local competition with strains with this strategy so that $w_{\text {res }}=$ $w\left(f_{\text {res }} \mid f_{\text {res }}\right)$. If strains in the local patch do not meet any other strain $(n=1)$, then the resident and migrant strategy's fitnesses are determined by their autonomous growth, following a single set of ODEs (Eq. 2). We define the fitness of a strain as its cell density at the end of a competition phase, which is after a fixed amount of competition time $t_{\text {end. }}$ We then compute the invasion index for an invading strategy as defined by Mitri et al. (2011), 


$$
I_{\text {inv }}=\frac{w_{\text {inv }}}{w_{\text {res }}}=\frac{w\left(f_{\text {inv }} \vee f_{\text {res }}\right)}{w\left(f_{\text {res }} \vee f_{\text {res }}\right)}
$$

281 When the invasion index of a new strategy is larger than one $\left(I_{\mathrm{inv}}>1\right)$ the migrant strategy

will increase its meta-population frequency from its initial appearance to the next mixing step. When the strategy's invasion index is smaller than one $\left(I_{\mathrm{inv}}<1\right)$, it will go extinct. An important nuance of evolutionary invasion analysis is that a strategy's evolutionary success is not determined solely by its local competitive success: a migrant strategy that wins in a local patch against the resident strategy could still go extinct from the meta-population if the fitness of the resident strategy is high $\left(I_{\mathrm{inv}}<1\right)$. For example, a really aggressive strategy might win locally but harm itself so much in the process that it cannot outcompete the other patches in the meta population (Hauert et al. 2002).

We use the invasion index to follow the evolution of siderophore production and find optimal strategies. A successfully invading strategy will become the new resident strategy. This will occur repeatedly until we find a evolutionarily stable strategy (ESS, Maynard Smith 1982), which cannot itself be replaced by other strategies. Specifically, the ESS is a strategy that, if adopted by the entire population, cannot be invaded by any other strategy. We find ESSs by following the gradual evolution of strategies as they compete with others that are different to themselves. We test invading strategies that are locally-similar as well as strategies from the full range of possible strategies. By combining local and global searches in this way, we identify strategies that are evolutionarily stable in the face of a vast range of 299 possible competing strategies. While multiple ESS are theoretically possible in game theory, 300 we always found a unique ESS for each analysis. 


\section{$301 \quad$ Results}

302 Overview

303 We can use our eco-evolutionary model to study how siderophore production evolves across

304 different scenarios and, in particular, in response to siderophore privatisation. This allows us to test a range of ecologies where differing number of strains meet and compete for resources and we can vary the extent to which the different strains can share each other's siderophores.

307 We use this to identify the evolutionarily stable investment into siderophore production for each situation. We then study the physiological regulation of siderophore production and find that the regulatory strategies that evolve also depend strongly on how much siderophores are shared. Specifically, with siderophore privatisation our model predicts that production should increase when strains encounter competitors.

Privatisation strongly affects the evolution of siderophore production

314 Evolutionary studies typically treat siderophores as a canonical public good that benefits all

315 cells in an environment equally, where non-producers (cheaters) can thrive in the presence of siderophore producers (Griffin et al. 2004; West et al. 2006, 2007; Ross-Gillespie et al. 2007;

317 Brown et al. 2009; Nadell et al. 2009). The potential for one genotype to exploit another, however, rests upon strains mixing such that non-producers have access to the siderophore

319 producers. As such, a key prediction of the standard social evolution model of siderophores is that the evolution of production will decrease with an increasing number of strains mixing in local competition (Harrison et al. 2008). In social evolution terminology, the investment into cooperation will evolve to decrease under conditions of decreased relatedness (Hamilton 1964; Frank 1998).

We compare the evolutionarily stable siderophore production in the absence of competitors ( $n=1$, Figure 2, black diamond) and in the presence of increasing numbers of 
competitor strains. When siderophores are entirely shared $(s=1)$ and their benefit returns to the producer as much as to other cells, we recapitulate the classic social evolution prediction

330 siderophore sharing between genotypes $(s<1)$.

331

332

333

334

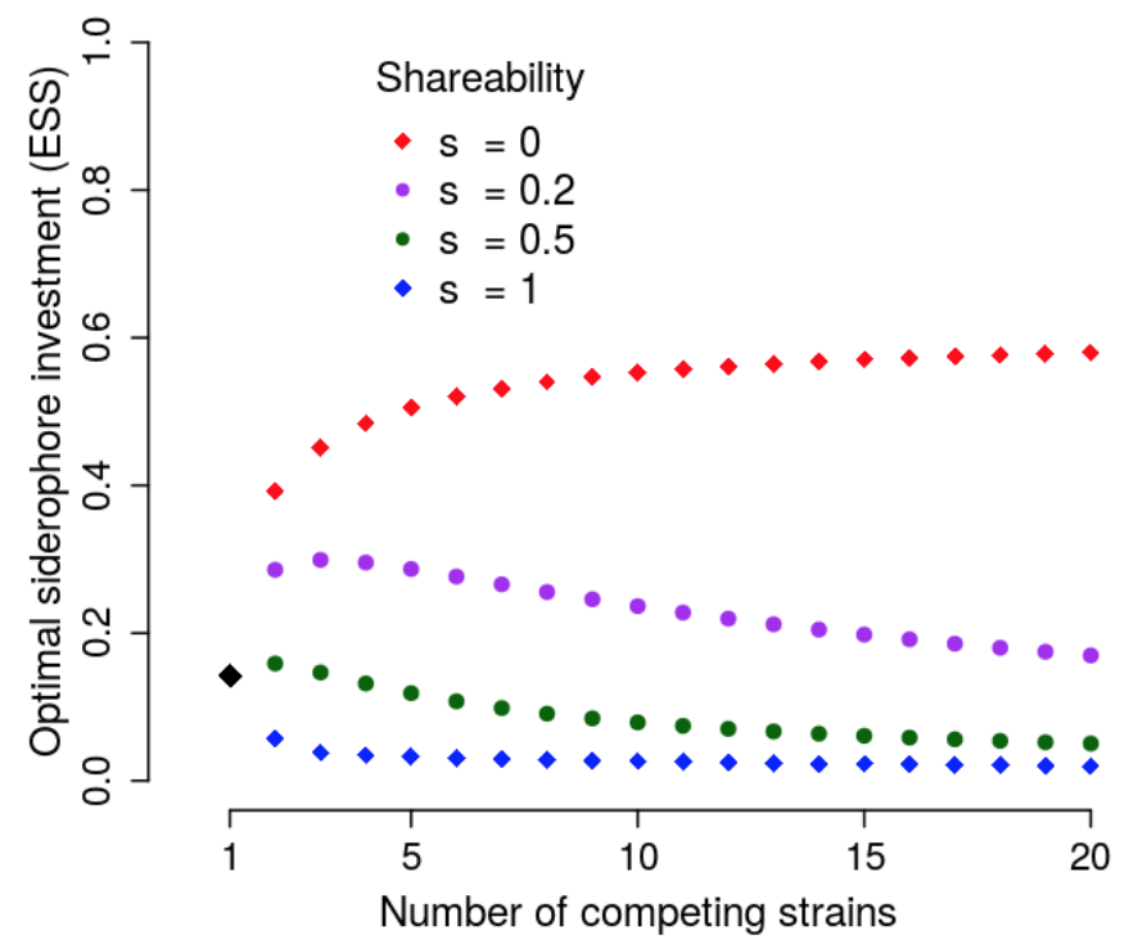

336 Figure 2 | The effect of competition and siderophore sharing on siderophore production. Using an adaptive dynamics frame work we find evolutionarily stable level of siderophore production for different levels of competition and siderophore sharing. We plot the optimal investment into siderophores $\left(f^{*}\right)$ against different numbers of competitors $(n)$ per local competition. In the absence of competition between strains, where siderophore sharing does not occur, the optimal investment into siderophore production is shown by the large black diamond on the far left. The effect of added competition on investment $\left(f^{*}\right)$ is qualitatively different for different levels of between-strain sharing of siderophores. When the number of competing strains increases, the production of an entirely shared siderophore is reduced (blue diamonds), while the production of a private siderophore is increased 
(red diamonds). At intermediate levels of sharing siderophore production first increases and then decreases with increasing competition (purple and green dots).

The privatisation of siderophores fundamentally changes the effects of strain mixing on production. When siderophores are private to the producer strain, increasing the intensity of competition between strains (increasing $n$ ) results in an increase in siderophore production (Figure 2). When siderophores are partly private and partly public, we see that the effect of increasing competition has elements of both the purely public and private evolutionary responses. Importantly, for relatively low levels of strain mixing, which may often be common due to spatial structuring in communities (Hallatschek et al. 2007; Oliveira et al. 2014; Mitri et al. 2015; Nadell et al. 2016), the effect of increasing competition is to increase siderophore investment (Figure 2). Siderophores are evolving as competitive traits. Even though siderophores are still a public good with respect to the cells of a single genotype, therefore, privatisation shifts them to behaving as a mechanism of exploitative competition, which is used to deplete and steal the iron of competitors.

\section{The effects of privatisation are robust for a wide range of conditions}

We have shown that privatisation can have strong effects on the evolution of siderophore production. In particular, privatisation means that siderophores evolve as a competitive rather than a cooperative trait, with investment increasing under conditions of high strain mixing (low relatedness). How robust is this effect? Our model contains a number of parameters that can be used to study how key ecological and biological factors influence the evolution of siderophore production. We performed sweeps of these parameters and studied in each case how strain mixing affects the evolved level of siderophore production (Figure 3). 

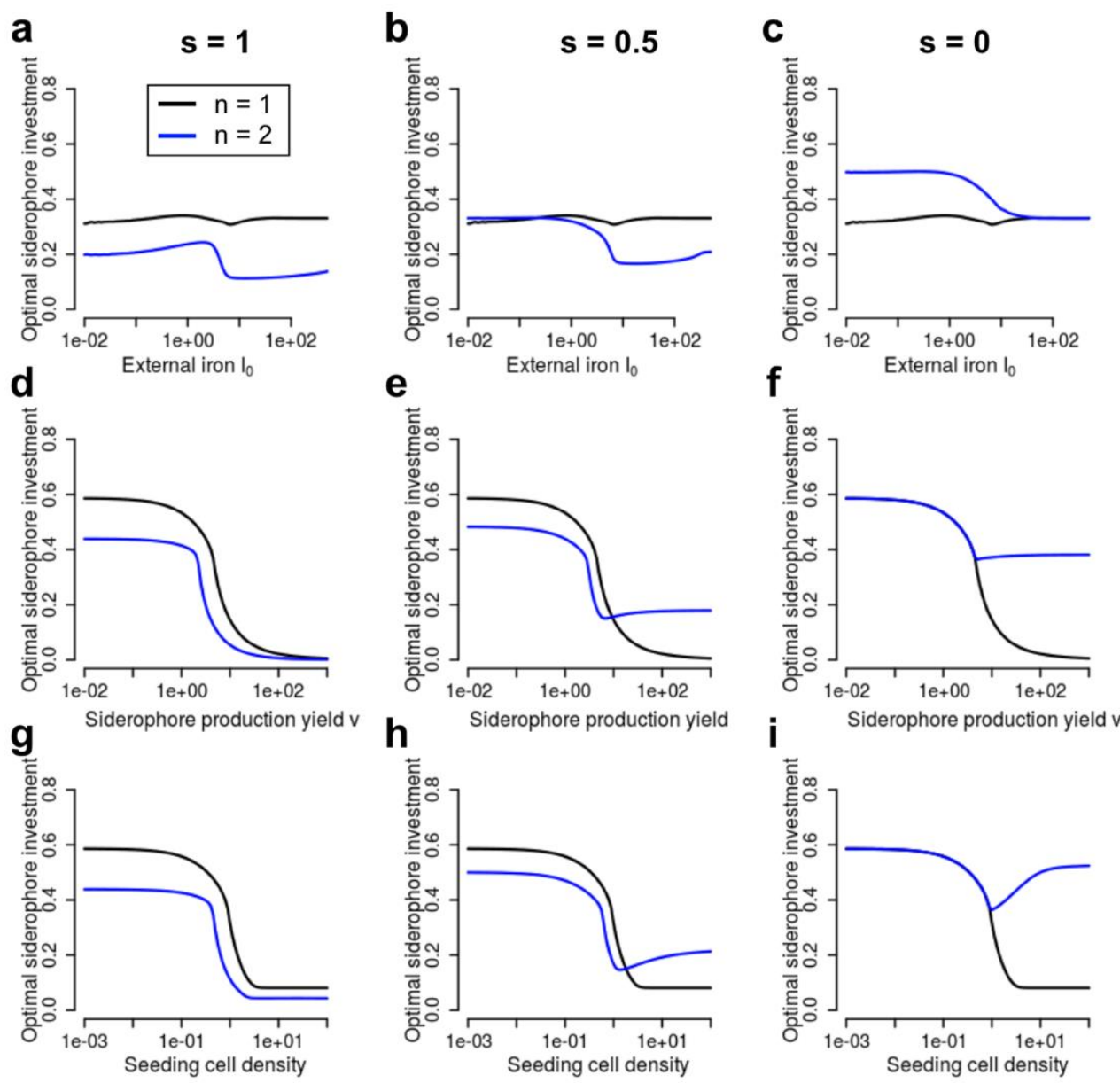

374 Figure 3 | Wide-ranging parameter sweeps show consistent effects of privatisation on

375 siderophore evolution. We study the effect of iron concentration, siderophore yield, and seeding cell density on ESS production of siderophores. We plot the optimal siderophore investment with and without competition ( $n=2, n=1$, respectively) over a range of external iron concentrations (a-c), and over a range of siderophore production yields (d-f), and over a range of seeding cell densities (g-i). We find that when siderophores are fully shared between strains $(s=1)$, adding local competition always decreases the evolutionarily stable level of siderophore production (a,d,g; blue line below black line). By contrast, for entirely private siderophores $(s=0)$, the production level increases $(\mathbf{c}, \mathbf{f}, \mathbf{i}$; blue line above black line). Under intermediate sharing ( $s=0.5)$ we observe regions of both decreased and increased siderophore production (b,e,h; crossings of blue and black line). When siderophores are mainly powerless to exploit the environment (high iron, low production yield and/or cell density) the cross-feeding effect dominates and production is reduced with strain mixing. But when siderophores become effective (low iron, high production yield and/or cell density) then their exploitative potential means that strains increase production in strain mixes. 
390 Our model is relatively complex in that it combines a dynamic model of local competitions

391 between strains, with global competition in a meta-population and search algorithms that

392 identify the evolutionarily stable strategy for each set of parameters. The predicted effects of

393 parameters on siderophore production are in some cases also relatively complex, with regions

394 where the ESS level of production decreases before increasing again (Figure 3). Nevertheless,

395 we observe clear and consistent effects of strain mixing on production level. When

396 siderophores are fully public $(s=1)$, introducing local competition between strains (increased

397 mixing) always decreases the evolutionarily stable level of siderophore production (Figure 3;

398 blue line below black line).

399 By contrast, with no sharing of siderophore between strains $(s=0)$, the investment

400 level increases with adding a competitor (Figure 3; blue line above black line). For some

401 parameters, this decrease is minor, particularly when siderophore production becomes

402 minimal (low production yield or seeding cell density) or when iron is abundant, because

403 under these conditions siderophores have little effect on competitors and are produced

404 similarly to the single strain case. However, critically, for private siderophores we never see a

405 decrease, and for public siderophores, we never see an increase in production when we move

406 from the clonal group to the mixed-genotype group (Figure 3).

407 Conditions of intermediate sharing $(s=0.5)$ are again intermediate in their behaviour

408 and we see that, dependent on parameters, for all three parameters strain mixing can drive an

409 increase or decrease in the evolutionarily stable level of siderophore production. Essentially,

410 in conditions where siderophores are powerless to exploit competitors (high iron, low

411 production yield and/or cell density) the effect of cross-feeding siderophore dominates and,

412 hence, its production is reduced with strain mixing. But when siderophores are efficient (low

413 iron, high production yield and/or cell density) the benefit from exploiting can overcome the 
414 effect of cross-feeding (Figure 3; crossing of black and blue line) so that production is 415 increased in strain mixes.

416 In summary, privatisation has strong and consistent effects on the evolution of

417 siderophore production that are robust for a wide range of ecological and biological

418 conditions.

419

420

Privatisation is critical to the evolution of siderophore regulation

421 As is typical of previous theoretical work on siderophore evolution (West and Buckling 2003;

422 Lee et al. 2012, 2016), we have so far treated siderophore production as a constitutive trait where each cell invests a fixed proportion of its resources $(f)$ into siderophores. However, in reality siderophores are often strongly regulated in response to environmental conditions (Harrison et al. 2008; Kümmerli et al. 2009b). We therefore extend our model to consider regulation of siderophore production and evolution of this regulation. We study regulation based on three sources of information in the environment, which are known to affect bacterial regulatory networks for multiple traits: iron concentration (Ratledge and Dover 2000; Wandersman and Delepelaire 2004), density of clone mate cells (e.g. quorum-sensing) (Waters and Bassler 2005), and density of competitor cells (Abrudan et al. 2015) (e.g. competition sensing (Cornforth and Foster 2013)) (Methods). For each type of sensing, we can then follow the evolution of strategies as before and identify the evolutionarily stable strategies of regulation (Methods, Figure 4). 
Private siderophores $(s=0)$
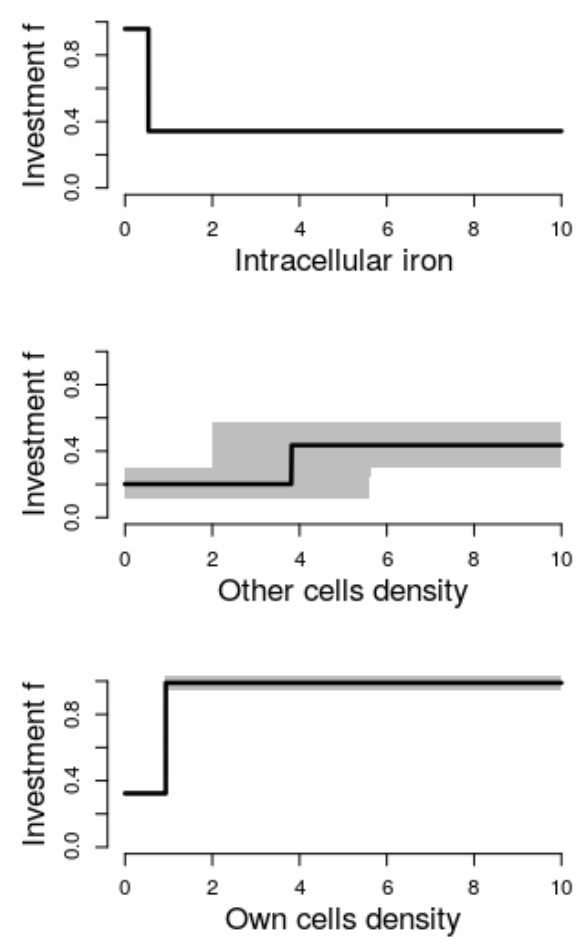

Public siderophores $(s=1)$
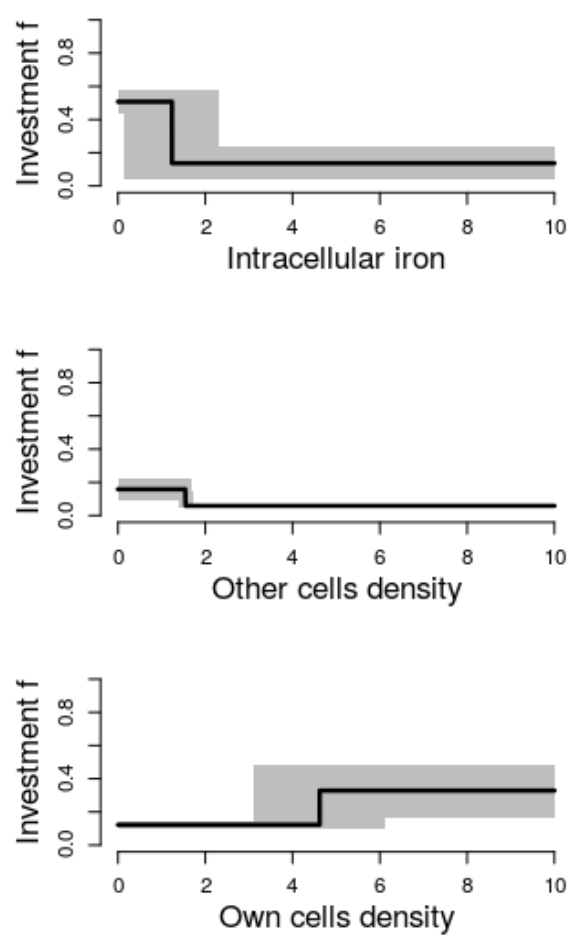
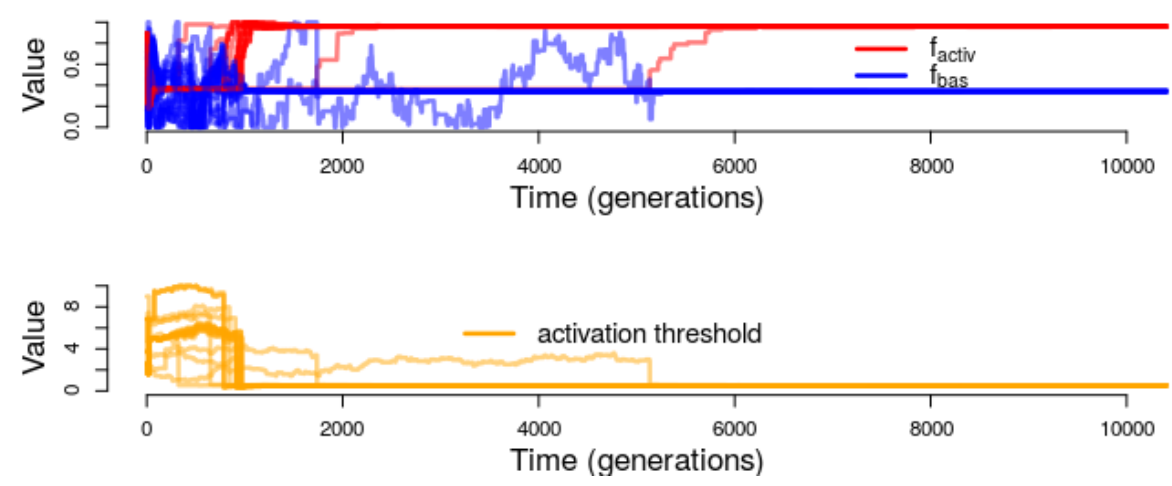

Figure 4 | The evolution of physiological siderophore regulation. We evolve the sensing function for siderophore production in pairwise competitions $(n=2)$ using the evolutionary algorithm outlined in Supplementary Methods and Results. We study three cases that each capture a different source of information: intracellular iron, own cell density and competitor cell density. The plots show the evolved response in siderophore production as a function of the sensed signal, as a mean from 30 runs of the simulation. The grey area shows the standard deviation. Siderophores evolve to be upregulated at low iron, independent of the shareability $(\mathbf{a}, \mathbf{b})$, consistent with siderophores being most valuable when iron is low. Siderophores evolve to be upregulated for high quorum (c,d) consistent with their efficiency being highest at high cell density. However, when siderophores are regulated based upon the density of competing cells, they are downregulated when siderophores are shared $(s=1)$ and upregulated when siderophores are private $(s=0)(\mathbf{e}, \mathbf{f})$. The bottom two plots show illustrative timeplots from the evolutionary algorithm, showing how the initial, and the activated siderophore investment $(\mathbf{g})$ and the activation threshold $(\mathbf{h})$ evolve in 30 realisations of the algorithm. The examples shown are for the evolution of quorum sensing and $s=0$. 
455 Siderophore regulation in response to iron level and own cell density evolve in a consistent way, irrespective of the degree of privatisation. The responses evolve such that low iron and high quorum both favour the release of siderophore. There is a clear post-hoc logic to these forms of regulation. Most simply, siderophores are most valuable when iron is low and our predicted regulation is well supported by empirical work that shows that in a number of

460 different species siderophore production can be strongly upregulated by low iron (Schmitt and Holmes 1991; Ratledge and Dover 2000; Rodriguez et al. 2002; Chakraborty et al. 2013).

The quorum-based regulation recapitulates the typical interpretation of the evolutionary function of quorum sensing. Quorum sensing allows a group of cells to only release a secreted product once the cell density of the focal strain is high enough to generate and receive an effective concentration of the product (Schluter et al. 2016). Consistent with this prediction, there is evidence that siderophore production increases at high cell density for a number of species (Stintzi et al. 1998; Lewenza et al. 1999; Mok et al. 2003). competing cells, we see a different pattern. Now, whether cells upregulate or downregulate 470 production depends upon whether siderophores are public or private (Figure $4 \mathrm{e}, \mathrm{f}$ ). The evolution of the regulation of public siderophores leads to a response where production is down regulated in response to increased numbers of the competing strain. This is because under these conditions, increased competition means that there is a greater threat of

474 siderophore piracy from the competitors and so downregulation benefits the focal strain.

475 When siderophores are privatised, we predict the opposite pattern. Now, siderophores 476 respond as expected for a competitive trait, such as the release of an antibiotic that kills 477 another strain (Cornforth and Foster 2013), with secretion upregulated in response to the 478 presence of the competing strain. 

known regulatory responses to iron level (Schmitt and Holmes 1991; Ratledge and Dover 2000; Rodriguez et al. 2002; Chakraborty et al. 2013) and quorum sensing (Stintzi et al. 1998; Lewenza et al. 1999; Mok et al. 2003). However, we see the same predictions for 483 private and public siderophores so the data are unable to distinguish between the two models. 484 By contrast, the predicted regulation based upon the level of competition with other strains changes depending on whether siderophores are private or public. This latter form of regulation, therefore, lends itself to testing the importance of privatisation for the evolution of siderophore use.

$$
\text { Does siderophore production, therefore, increase or decrease upon strain mixing? }
$$

489 Empirical work suggests that siderophore production typically increases in the presence of unrelated strains and species. Specifically, co-culture experiments between $P$. aeruginosa with Staphylococcus aureus found that $P$. aeruginosa makes more siderophores in the presence of S. aureus (Harrison et al. 2008). More recently, Traxler et al. (2013) placed colonies of Streptomyces species either alone, or next to a colony of a different species. When next to a foreign colony, the Streptomyces strain increased secretion of a number of siderophores (Traxler et al. 2013). Caution is required as the underlying mechanisms driving the upregulation of siderophore production is not yet clear and the responses may be driven

497 by multiple factors, including iron limitation. Nevertheless, the data in both cases are most 498 consistent with the upregulation of siderophores upon competition with other species, not the 499 downregulation expected for a public good. 


\section{Discussion}

502 Siderophores have emerged as a powerful model system to understand microbial sociality

503 (Griffin et al. 2004; Ross-Gillespie et al. 2007; Buckling and Brockhurst 2008; Kümmerli et

504 al. 2009a; Ross-Gillespie et al. 2009; Luján et al. 2015). In mixed cultures with a wildtype

505 producer strain, siderophore null mutants (cheaters) can thrive and outcompete the wildtype,

506 which is consistent with the idea that siderophores act as canonical public goods in microbial

507 communities. This view was recently emphasised in discussions of "black queen" evolution

508 (Oliveira et al. 2014; Morris 2015). Microbes may commonly lose genes, including those for

509 siderophores, when they can be complemented by other strains and species in their diverse

510 communities (Cordero et al. 2012; Andersen et al. 2015). However, it is also clear that the

511 siderophores of one strain are often not fully shared with other strains, because of the use of

512 specific receptors to import siderophore (Joshi et al. 2006; Khan et al. 2006; Lee et al. 2012),

513 and limited diffusion (Nadell et al. 2010; Julou et al. 2013; Kümmerli et al. 2014).

$514 \quad$ Here we have shown how limited siderophore sharing between strains has

515 fundamental effects on their ecology and evolution, which are missed in the traditional public

516 goods model. With privatisation, strains that face a lot of competition from other genotypes

517 are expected to evolve to increase their investment in siderophores (Figure 2), rather than the

518 decrease expected from a public good. We also find that the effects of privatisation are

519 mirrored in the evolution of siderophore regulation. When siderophores are fully shared, our

520 model predicts that cells will evolve to downregulate production when competing strains are

521 detected. By contrast, when siderophores are privatised, regulation evolves to increased

522 siderophore production in the presence of competing strains: siderophores then function as a

523 way to compete with other genotypes (Figure 4); in particular, they become mediators of

524 exploitative competition (Hibbing et al. 2010). 

presence of other strains, consistent with it being used in competition (Harrison et al. 2008; Traxler et al. 2013). More generally, there is growing evidence that bacteria are capable of regulating a wide range of traits based upon the presence of different strains. Competing genotypes can be detected by quorum sensing autoinducers or other molecules released into

530 the environment (Keller and Surette 2006; Cornforth and Foster 2013; LeRoux et al. 2015).

531 Another way to achieve detection is via competition sensing, in particular via stress responses that detect the cell damage caused by the toxins of competing strains (Basler and Mekalanos 2012; Cornforth and Foster 2013). The discussion of competitive responses has so far focused on bacterial warfare and the upregulation of toxins and type VI secretion systems in response to ecological competition. However, siderophores are another potential way to compete with other genotypes, as is biofilm formation, which was also recently found to be upregulated in response to competition (Oliveira et al. 2015). dominate eco-evolutionary dynamics whenever competition is primarily between cells with a

540 recent common ancestor, as may occur in chronic diseases like cystic fibrosis (Andersen et al. 541 2015). However, the ecology of many bacterial species centres upon competition in diverse communities, where strain-specific siderophores limit between genotype sharing (Joshi et al.

543 2006; Khan et al. 2006). We have shown that these conditions strongly affect how

544 siderophores function in nature. Siderophores are no longer a simple public good. Instead, 545 siderophores become a competitive phenotype that, like antibiotics, is upregulated in order to overcome other strains. 
550

551

552

553

554

555

556

557

558

559

560

561

562

563

564

565

566

567

568

569

570

571

572

573

574

575

576

577

578

579

580

581

582

583

584

585

586

587

588

589

590

591

592

Abrudan, M. I., F. Smakman, A. J. Grimbergen, S. Westhoff, E. L. Miller, G. P. van Wezel, and D. E. Rozen. 2015. Socially mediated induction and suppression of antibiosis during bacterial coexistence. Proc. Natl. Acad. Sci. 201504076.

Andersen, S. B., R. L. Marvig, S. Molin, H. Krogh Johansen, and A. S. Griffin. 2015. Longterm social dynamics drive loss of function in pathogenic bacteria. Proc. Natl. Acad. Sci. 201508324.

Andrews, S. C., A. K. Robinson, and F. Rodríguez-Quiñones. 2003. Bacterial iron homeostasis. FEMS Microbiol. Rev. 27:215-237.

Basler, M., and J. J. Mekalanos. 2012. BREVIA Type 6 Secretion Dynamics Within and Between Bacterial Cells. Science 337:2115. American Association for the Advancement of Science.

Boukhalfa, H., and A. L. Crumbliss. 2002. No Title. BioMetals 15:325-339. Kluwer Academic Publishers.

Braun, V. 2001. Iron uptake mechanisms and their regulation in pathogenic bacteria. Int. J. Med. Microbiol. 291:67-79.

Brown, S. P., S. A. West, S. P. Diggle, and A. S. Griffin. 2009. Social evolution in microorganisms and a Trojan horse approach to medical intervention strategies. Philos. Trans. R. Soc. B Biol. Sci. 364:3157-3168.

Buckling, A., and M. A. Brockhurst. 2008. Kin selection and the evolution of virulence. Heredity (Edinb). 100:484-488.

Carson, K. C., A. R. Glenn, and M. J. Dilworth. 1994. Specificity of siderophore-mediated transport of iron in rhizobia. Arch. Microbiol. 161:333-339. Springer-Verlag.

Chakraborty, R., V. Braun, K. Hantke, and P. Cornelis (eds). 2013. Iron Uptake in Bacteria with Emphasis on E. coli and Pseudomonas. Springer Netherlands, Dordrecht.

Cordero, O. X., L. -a. Ventouras, E. F. DeLong, and M. F. Polz. 2012. Public good dynamics drive evolution of iron acquisition strategies in natural bacterioplankton populations. Proc. Natl. Acad. Sci. 109:20059-20064.

Cornelis, P. 2010. Iron uptake and metabolism in pseudomonads.

Cornelis, P., and J. Bodilis. 2009. A survey of TonB-dependent receptors in fluorescent pseudomonads. Environ. Microbiol. Rep. 1:256-62.

Cornelis, P., D. Hohnadel, and J. M. Meyer. 1989. Evidence for different pyoverdinemediated iron uptake systems among Pseudomonas aeruginosa strains. Infect. Immun. 57:3491-3497.

Cornelis, P., and S. Matthijs. 2002. Diversity of siderophore-mediated iron uptake systems in fluorescent pseudomonads: not only pyoverdines. Environ. Microbiol. 4:787-798.

Cornforth, D. M., and K. R. Foster. 2013. Competition sensing: the social side of bacterial stress responses. Nat. Rev. Microbiol. 11:285-93. Nature Publishing Group.

Cremer, J., A. Melbinger, and E. Frey. 2012. Growth dynamics and the evolution of cooperation in microbial populations. Sci. Rep. 2:281.

Crowley, D. E., Y. C. Wang, C. P. P. Reid, and P. J. Szaniszlo. 1991. Mechanisms of iron acquisition from siderophores by microorganisms and plants. Plant Soil 130:179-198.

Dumas, Z., A. Ross-Gillespie, and R. Kummerli. 2013. Switching between apparently redundant iron-uptake mechanisms benefits bacteria in changeable environments. Proc Biol Sci 280:20131055.

Eberl, H. J., and S. Collinson. 2009. A modeling and simulation study of siderophore mediated antagonism in dual-species biofilms. Theor. Biol. Med. Model. 6:30.

Fgaier, H., and H. J. Eberl. 2010. A competition model between Pseudomonas fluorescens and pathogens via iron chelation. J. Theor. Biol. 263:566-578. Elsevier. 
Frank, S. S. A. 1998. Foundations of social evolution. Princeton University Press, New Yersey.

Griffin, A. S., S. A. West, and A. Buckling. 2004. Cooperation and competition in pathogenic bacteria. Nature 430:1024-1027.

Hallatschek, O., P. Hersen, S. Ramanathan, and D. R. Nelson. 2007. Genetic drift at expanding frontiers promotes gene segregation. Proc. Natl. Acad. Sci. U. S. A. 104:19926-30.

Hamilton, W. D. 1964. The genetical evolution of social behaviour. I. J. Theor. Biol. Hantke, K. 2001. Iron and metal regulation in bacteria. Curr. Opin. Microbiol. 4:172-177.

Harrison, F., J. Paul, R. C. Massey, and A. Buckling. 2008. Interspecific competition and siderophore-mediated cooperation in Pseudomonas aeruginosa. ISME J. 2:49-55. International Society for Microbial Ecology.

Hauert, C., S. De Monte, J. Hofbauer, and K. Sigmund. 2002. Volunteering as Red Queen mechanism for cooperation in public goods games. Science 296:1129-32.

Hibbing, M. E., C. Fuqua, M. R. Parsek, and S. B. Peterson. 2010. Bacterial competition: surviving and thriving in the microbial jungle. Nat. Rev. Microbiol. 8:15-25.

Hider, R. C., and X. Kong. 2010. Chemistry and biology of siderophores. Nat. Prod. Rep. 27:637. The Royal Society of Chemistry.

Inglis, R. F., P. G. Roberts, A. Gardner, and A. Buckling. 2011. Spite and the scale of competition in Pseudomonas aeruginosa. Am. Nat. 178:276-285.

Joshi, F., G. Archana, and A. Desai. 2006. Siderophore cross-utilization amongst rhizospheric bacteria and the role of their differential affinities for $\mathrm{Fe} 3+$ on growth stimulation under iron-limited conditions. Curr. Microbiol. 53:141-147.

Julou, T., T. Mora, L. Guillon, V. Croquette, I. J. Schalk, D. Bensimon, and N. Desprat. 2013. Cell-cell contacts confine public goods diffusion inside Pseudomonas aeruginosa clonal microcolonies. Proc. Natl. Acad. Sci. U. S. A. 110:12577-82.

Keller, L., and M. G. Surette. 2006. Communication in bacteria: an ecological and evolutionary perspective. Nat. Rev. Microbiol. 4:249-58. Nature Publishing Group.

Khan, A., R. Geetha, A. Akolkar, A. Pandya, G. Archana, and A. J. Desai. 2006. Differential cross-utilization of heterologous siderophores by nodule bacteria of Cajanus cajan and its possible role in growth under iron-limited conditions. Appl. Soil Ecol. 34:19-26.

Kraemer, S. M. 2004. Iron oxide dissolution and solubility in the presence of siderophores. Aquat. Sci. 66:3-18.

Kümmerli, R., A. Gardner, S. a. West, and A. S. Griffin. 2009a. Limited dispersal, budding dispersal, and cooperation: An experimental study. Evolution (N. Y). 63:939-949.

Kümmerli, R., N. Jiricny, L. S. Clarke, S. a. West, and a. S. Griffin. 2009b. Phenotypic plasticity of a cooperative behaviour in bacteria. J. Evol. Biol. 22:589-598.

Kümmerli, R., K. T. Schiessl, T. Waldvogel, K. McNeill, and M. Ackermann. 2014. Habitat structure and the evolution of diffusible siderophores in bacteria. Ecol. Lett. 17:15361544.

Lee, W., M. van Baalen, and V. A. A. Jansen. 2012. An evolutionary mechanism for diversity in siderophore-producing bacteria. Ecol. Lett. 15:119-125.

Lee, W., M. van Baalen, and V. A. A. Jansen. 2016. Siderophore production and the evolution of investment in a public good: An adaptive dynamics approach to kin selection. J. Theor. Biol. 388:61-71. Elsevier.

LeRoux, M., S. B. Peterson, and J. D. Mougous. 2015. Bacterial danger sensing. J. Mol. Biol. 1-10. Elsevier B.V.

Lewenza, S., B. Conway, E. P. Greenberg, and P. A. Sokol. 1999. Quorum Sensing in Burkholderia cepacia: Identification of the LuxRI Homologs CepRI. J. Bacteriol. 181:748-756. 
662

663

664

665

666

667

668

669

670

671

672

673

674

675

676

677

678

679

680

681

682

683

684

685

686

687

688

689

690

691

692

693

694

695

696

697
Luján, A. M., P. Gómez, and A. Buckling. 2015. Siderophore cooperation of the bacterium Pseudomonas fluorescens in soil. Biol. Lett. 11:20140934.

Maynard Smith, J. 1982. Evolution and the Theory of Games.

Mey, A. R., J. H. Crosa, and S. M. Payne. 2004. Iron Transport in Bacteria. American Society of Microbiology.

Miethke, M., and M. A. Marahiel. 2007. Siderophore-based iron acquisition and pathogen control. Microbiol. Mol. Biol. Rev. 71:413-51.

Mitri, S., E. Clarke, and K. R. Foster. 2015. Resource limitation drives spatial organization in microbial groups. ISME J. 1-12. Nature Publishing Group.

Mitri, S., J. B. Xavier, and K. R. Foster. 2011. Social evolution in multispecies biofilms. Proc. Natl. Acad. Sci. 108(Suppl 2):10839-10846.

Mok, K. C., N. S. Wingreen, and B. L. Bassler. 2003. Vibrio harveyi quorum sensing: a coincidence detector for two autoinducers controls gene expression. EMBO J. 22:87081. EMBO Press.

Morris, J. J. 2015. Black Queen evolution: the role of leakiness in structuring microbial communities. Trends Genet. 31:475-482.

Nadell, C. D., K. Drescher, and K. R. Foster. 2016. Spatial structure, cooperation, and competition in biofilms. Nat. Rev. Microbiol. accepted.

Nadell, C. D., K. R. Foster, and J. B. Xavier. 2010. Emergence of Spatial Structure in Cell Groups and the Evolution of Cooperation. PLoS Comput. Biol. 6:e1000716. Public Library of Science, Department of Ecology and Evolutionary Biology, Princeton University, Princeton, New Jersey, United States of America.

Nadell, C. D., J. B. Xavier, and K. R. Foster. 2009. The sociobiology of biofilms. FEMS Microbiol. Rev. 33:206-224.

Nowak, M. A., and K. Sigmund. 2004. Evolutionary dynamics of biological games. Science 303:793-9. American Association for the Advancement of Science.

Oliveira, N. M., E. Martinez-Garcia, J. Xavier, W. M. Durham, R. Kolter, W. Kim, and K. R. Foster. 2015. Biofilm Formation As a Response to Ecological Competition. PLOS Biol. 13:e1002191.

Oliveira, N. M., R. Niehus, and K. R. Foster. 2014. Evolutionary limits to cooperation in microbial communities. Proc. Natl. Acad. Sci. 111:201412673.

Ratledge, C., and L. G. Dover. 2000. Iron metabolism in pathogenic bacteria. Annu. Rev. Microbiol. 54:881-941.

Rodriguez, G. M., M. I. Voskuil, B. Gold, G. K. Schoolnik, and I. Smith. 2002. ideR, an Essential Gene in Mycobacterium tuberculosis: Role of IdeR in Iron-Dependent Gene Expression, Iron Metabolism, and Oxidative Stress Response. Infect. Immun. 70:33713381.

Ross-Gillespie, A., A. Gardner, A. Buckling, S. A. West, and A. S. Griffin. 2009. Density dependence and cooperation: theory and a test with bacteria. Evolution (N. Y). 63:23152325.

Ross-Gillespie, A., A. Gardner, S. A. West, and A. S. Griffin. 2007. Frequency Dependence and Cooperation: Theory and a Test with Bacteria. Am. Nat. 170:331-342. [University of Chicago Press, American Society of Naturalists].

Schluter, J., A. P. Schoech, K. R. Foster, and S. Mitri. 2016. The Evolution of Quorum Sensing as a Mechanism to Infer Kinship. PLOS Comput. Biol. 12:e1004848. Public Library of Science.

Schmitt, M. P., and R. K. Holmes. 1991. Iron-dependent regulation of diphtheria toxin and siderophore expression by the cloned Corynebacterium diphtheriae repressor gene $\mathrm{dtxR}$ in C. diphtheriae C7 strains. Infect. Immun. 59:1899-1904.

Stacy, A., L. McNally, S. E. Darch, S. P. Brown, and M. Whiteley. 2015. The biogeography 
of polymicrobial infection. Nat. Rev. Microbiol. 14:93-105. Nature Publishing Group, a division of Macmillan Publishers Limited. All Rights Reserved.

Stintzi, A., K. Evans, J. Meyer, and K. Poole. 1998. Quorum-sensing and siderophore biosynthesis in Pseudomonas aeruginosa: lasRllasI mutants exhibit reduced pyoverdine biosynthesis. FEMS Microbiol. Lett. 166:341-345. The Oxford University Press.

Traxler, M. F., J. D. Watrous, T. Alexandrov, P. C. Dorrestein, and R. Kolter. 2013. Interspecies interactions stimulate diversification of the Streptomyces coelicolor secreted metabolome. MBio 4:1-12.

Wandersman, C., and P. Delepelaire. 2004. Bacterial Iron Sources: From Siderophores to Hemophores. Annu. Rev. Microbiol. 58:611-647.

Waters, C. M., and B. L. Bassler. 2005. Quorum Sensing : Communication in Bacteria. Annu. Rev. Cell Dev. Biol. 21:319-346.

West, S. A., and A. Buckling. 2003. Cooperation, virulence and siderophore production in bacterial parasites. Proc. Biol. Sci. 270:37-44.

West, S. A., S. P. Diggle, A. Buckling, A. Gardner, and A. S. Griffin. 2007. The Social Lives of Microbes. Annu. Rev. Ecol. Evol. Syst. 38:53-77.

West, S. A., A. S. Griffin, A. Gardner, and S. P. Diggle. 2006. Social evolution theory for microorganisms. Nat. Rev. Microbiol. 4:597-607.

Winkelmann, G. 1991. CRC handbook of microbial iron chelates. CRC Press.

Winkelmann, G., D. Van der Helm, and J. Neilands. 1987. Iron transport in microbes, plants, and animals. 


\section{Supplementary Methods and Results}

721 In this supplementary material, we describe how we obtain the local strain dynamics given

722 through equation (1) in the main text.

723

\section{Explicit interaction dynamics that lead to equation 1}

725

726

727

728

729

$$
\left\{\begin{array}{c}
\frac{d C}{d t}=\mu\left(\frac{(1-f) N}{(1-f) N+K}\left(\frac{P}{P+K_{P}}+\epsilon I\right)\right) C-d_{C} C \\
\frac{d S}{d t}=V f N C+\frac{(1-f) N}{(1-f) N+K} \frac{P}{P+K_{P}} C-\gamma S I-d_{S} S \\
\frac{d P}{d t}=\gamma S I-\frac{(1-f) N}{(1-f) N+K} \frac{P}{P+K_{P}} C-d_{P} P \\
\frac{d I}{d t}=a\left(I_{0}-I\right)-\gamma S I-\epsilon C I,
\end{array}\right.
$$

Starting from the assumptions described in the Methods section, we explicitly model the dynamics of siderophore scavenging within a single patch for a single-strain $(n=1)$ by the ODE system (\$1)

(n)

730

731

732

733 
740 limited and therefore constant nutrient $(N)$, the available iron-siderophore complex $(P)$ and

741 iron uptake through siderophore-independent mechanisms $(\varepsilon)$. In this first equation, we

742 explicitly model the presence of nutrient $N$ that represents the cells' source of energy. Cells

743 invest a fraction 1 - $f$ of their energy into biomass accumulation and the conversion of

744 nutrient into biomass is given by a saturating Monod function with saturation constant $K$. The

745 acquisition of iron through uptake of siderophore-iron complexes also follows a Monod

746 function with constant $K_{\mathrm{P}}$. The free siderophore concentration $S$ changes over time due to

747 cellular siderophore production, which is proportional to the fraction of non-limited nutrients

748 that the cells allocate to siderophore production $(f N)$ and to the siderophore production yield

$749(V)$. Siderophores are recycled from iron-siderophore complexes and are lost from the system

750 through diffusion $\left(d_{\mathrm{s}}\right)$. The concentration of iron-bound siderophores changes over time due

751 to the formation of such complexes (at a rate $\gamma$, that represents the affinity of siderophore for

752 iron), on the uptake by cells, and on a loss term with loss parameter $d_{P}$. Finally, the

753 concentration of available iron follows a chemostat dynamics with a reflux of external iron

754 and depletion through the formation of iron-siderophore complexes. As the level of non-

755 limited nutrient $N$ is constant, we can reduce the system by introducing the parameter $\beta=\frac{K}{N}$.

756 To simplify the expression and we rename the corrected siderophore production yield $v=$

$757 V N=V \frac{K}{\beta}$, yielding equation 1 of the main text. 


\begin{tabular}{|c|c|c|}
\hline Parameter & Biological significance & $\begin{array}{l}\text { Default } \\
\text { value }\end{array}$ \\
\hline$v$ & siderophore production yield & 5 \\
\hline$d_{S}$ & free siderophore loss & 0.5 \\
\hline$d_{C}$ & cell mortality & 0.2 \\
\hline$d_{P}$ & iron-siderophore complex loss & 0.5 \\
\hline$I_{0}$ & external iron concentration & 10 \\
\hline$a$ & external iron reflux rate & 0.2 \\
\hline$\mu$ & cell maximum growth rate & 0.8 \\
\hline$\beta$ & $\begin{array}{l}\text { normalized nutrient saturation constant: } \beta=\frac{K}{N} \\
\text { with } \mathrm{K} \text { the saturation constant of the Monod } \\
\text { function for nutrient-dependent growth and } \mathrm{N} \text { the } \\
\text { constant nutrient level }\end{array}$ & 1 \\
\hline$\rho$ & between-siderophore iron exchange rate & 0 \\
\hline$\epsilon$ & siderophore-independent iron capture rate & 0 \\
\hline$K_{P}$ & $\begin{array}{l}\text { Monod saturating constant for the uptake of iron- } \\
\text { siderophore complexes }\end{array}$ & 1 \\
\hline$\gamma$ & siderophore-iron binding affinity & 5 \\
\hline
\end{tabular}

760 Supplementary Table 1 | Biological significance and default values of the parameters used

761 in the model 